\title{
Status of vertex and tracking detector R\&D at CLIC
}

\author{
Elena FIRU ${ }^{1}$ \\ ${ }^{I}$ Institute of Space Science \\ Atomistilor 409 Bucharest, Romania \\ E-mail: efirulcern.ch \\ On behalf of the CLICdp collaboration
}

The physics aims at the future CLIC high-energy linear $\mathrm{e}^{+} \mathrm{e}^{-}$collider set very high precision requirements on the performance of the vertex and tracking detectors. Moreover, these detectors have to be well adapted to the experimental conditions, such as the bunch train structure of the beam and the presence of beam-induced backgrounds. The principal challenges are: a point resolution of a few micron, ultra-low mass $\left(\sim 0.2 \% \mathrm{X}_{0}\right.$ per layer for the inner vertex region), very low power dissipation (compatible with air-flow cooling in the inner vertex region) and pulsed power operation, complemented with $\sim 10 \mathrm{~ns}$ time stamping capabilities. An overview of the R\&D program for pixel and tracking detectors at CLIC will be presented, including recent results on an innovative hybridisation concept based on capacitive coupling between active sensors (HV-CMOS) and readout ASICs (CLICpix).

The European Physical Society Conference on High Energy Physics

22-29 July 2015

Vienna, Austria

${ }^{1}$ Speaker
( Copyright owned by the author(s) under the terms of the Creative Commons
Attribution-NonCommercial-NoDerivatives 4.0 International License (CC BY-NC-ND 4.0). 


\section{CLIC accelerator and detector}

The Compact Linear Collider (CLIC) is a project for an electron-positron collider with a centre-of- mass energy of up to $3 \mathrm{TeV}$. The experiment at the CLIC interaction-point is composed of an ultra-low-mass vertex detector, a silicon tracker, fine grained calorimeters, all located inside a superconducting solenoid providing a field of 4-5 T. Details of CLIC, its physics potential, the detector and its performance are described elsewhere [1].

\section{CLIC vertex detector requirements}

The vertex detector must fulfill stringent requirements. It should have:

- an efficient tagging of heavy quarks through precise determination of displaced vertices. Monte Carlo simulations show that these goals can be met in terms of the precision of the transverse impact-parameter

$$
\sigma\left(d_{0}\right)=\sqrt{a^{2}+b^{2} \cdot G e V^{2} /\left(p^{2} \sin ^{3} \theta\right)}
$$

if a high-momentum term of $\mathrm{a} \sim 5 \mu \mathrm{m}$ and a multiple scattering term of $\mathrm{b} \sim 15 \mu \mathrm{m}$ can be achieved ( $p$ is the momentum of the particle and $\theta$ is the polar angle with respect to the beam axis);

- a good single point resolution, $\sigma_{\mathrm{SP}} \sim 3 \mu \mathrm{m}$, that implies small pixels of approximately 25 $\mu \mathrm{m} \times 25 \mu \mathrm{m}$;

- low material budget, smaller than $0.2 \% \mathrm{X}_{\mathrm{o}}$ per detection layer (including supports, cables and electronics);

- low power ASICs with a total power consumption of less than $50 \mathrm{~mW} / \mathrm{cm}^{2}$, allowing airflow cooling;

- trigger-less readout and pulsed powering, possible at CLIC which operates with $156 \mathrm{~ns}$ long bunch trains separated by $20 \mathrm{~ms}$ and has low event rates;

- time stamping with $\sim 10 \mathrm{~ns}$ accuracy to reject background, implying the need for highresistivity sensors with fast readout.

\section{Sensor and readout $R \& D$ programme 3.1 Thin sensor assemblies}

Sensor assemblies using hybrid planar pixel technology (sensor + readout ASIC) are developed for the vertex detector. In this scheme planar silicon sensors and readout chips are bump-bonded together.

The goal for the CLIC vertex detector is to use $50 \mu \mathrm{m}$ thick sensors and $50 \mu \mathrm{m}$ thick ASICs. In total this accounts for around $50 \%$ of the material budget. The pixel size is foreseen to be $25 \mu \mathrm{m} \times 25 \mu \mathrm{m}$. This should enable charge sharing, which in combination with analog readout will improve the single-hit resolution. In order to reduce dead space and improve tiling, sensors will have thin edges and will make use of Through Silicon Via (TSV) technology, (see Figure 1 for illustration). This provides a vertical electrical connection through the chip, therefore removing the need for wire bonds.

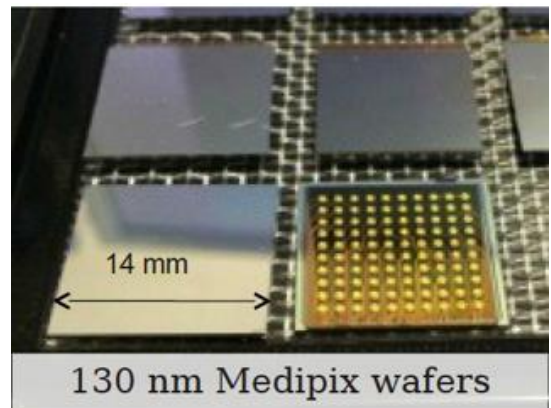

$130 \mathrm{~nm}$ Medipix wafers
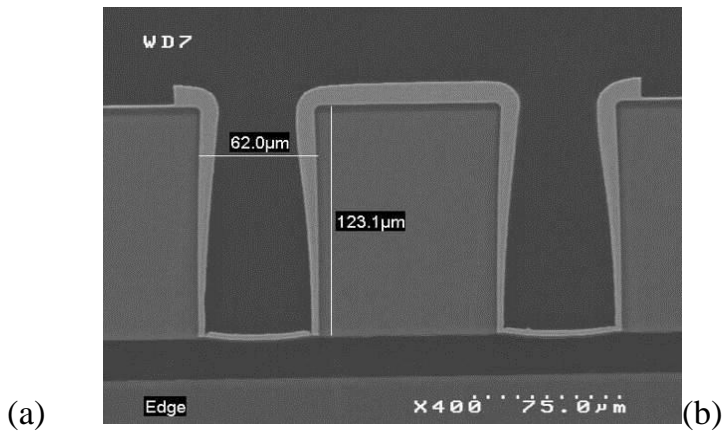

Figure 1: (a): Medipix $3 R X$ ASICs. The ASIC is processed with a redistribution layer for TSV connection. (b): Cut through a TSV connection inside a Medipix3RX ASIC from [2]. 


\subsection{Timepix beam tests}

The properties and performance of thin sensor assemblies have been investigated with test-beam measurements. A series of silicon sensors, with thickness between $50 \mu \mathrm{m}$ and $300 \mu \mathrm{m}$, were bonded to Timepix chips with $100 \mu \mathrm{m}$ and $700 \mu \mathrm{m}$ thickness. The pixel size of the assemblies was $55 \mu \mathrm{m}$ x $55 \mu \mathrm{m}$.

Test-beam measurements were performed on the assemblies at DESY and at the CERN PS [3]. In both places, the EUDET telescope [4], based on MIMOSA detectors, was used to provide track information with a position resolution at the Device Under Test (DUT) of $\sim 3 \mu \mathrm{m}$.

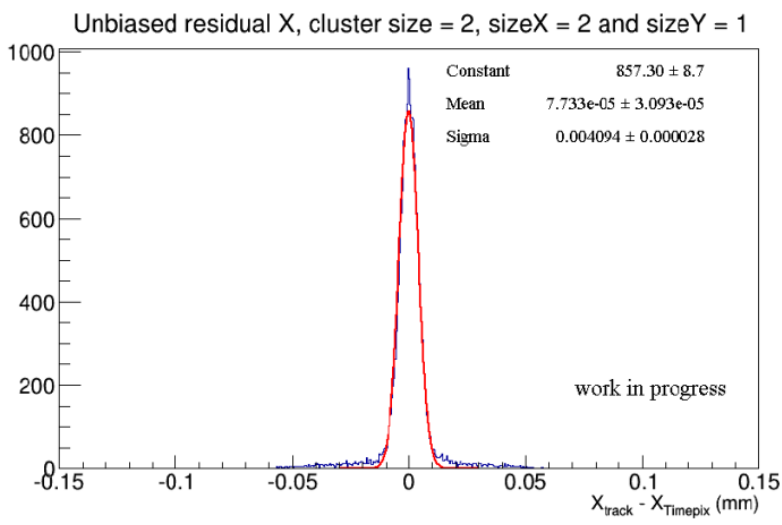

Figure 2: Residual distribution of two-hit cluster for a $50 \mu \mathrm{m}$ thick sensor

The test-beam analysis allows the measurement of the position residual of the thin sensor assemblies. Figure 2 shows the residual distribution for two-hit clusters in a $50 \mu \mathrm{m}$ thick sensor with $20 \mu \mathrm{m}$ wide active edges at a resolution $\sim 4.1 \mu \mathrm{m}$. The data was obtained with electrons of 5.6 GeV momentum. Multi-hit clusters are formed when the charge induced by a track is shared between neighboring pixels. Figure 3(a) shows the positions within the pixel of tracks which form one-hit clusters. In contrast, Figure 3(b) shows the positions of tracks which form two-hit clusters. The latter demonstrates that tracks forming two-hit clusters are located close to the border between pixels, as expected.

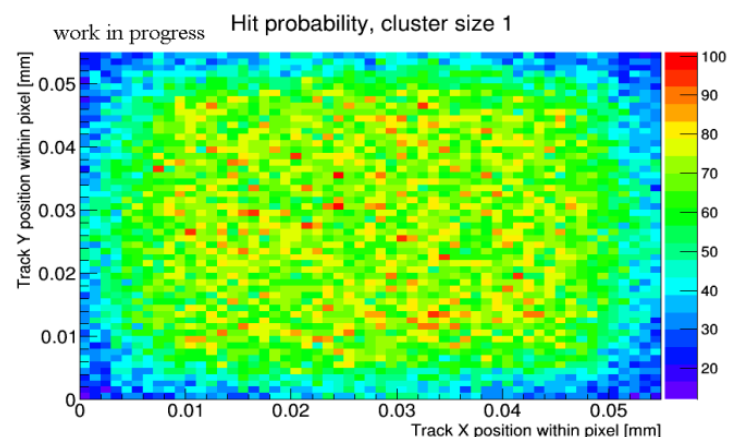

(a)

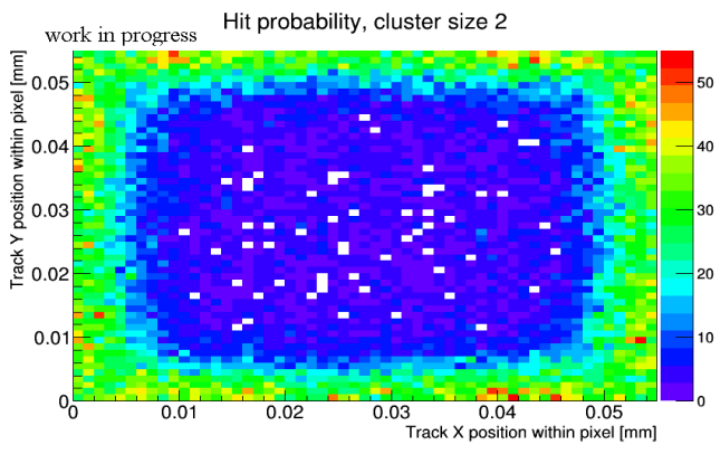

(b)

Figure 3: Reconstructed cluster position of cluster size 1 (a) and cluster size 2 (b) for a 50 بm thick sensor

\subsection{Timepix assembly calibration}

The Time-over-Threshold (TOT) energy measurement of the Timepix assemblies was calibrated based on the response to photons of known energy. For each photon energy the TOT response of the sensor is determined. The resulting data points are fit with a surrogate function, as illustrated in Figure 4. Details on the calibration of Timepix assemblies can be found in [5]. 

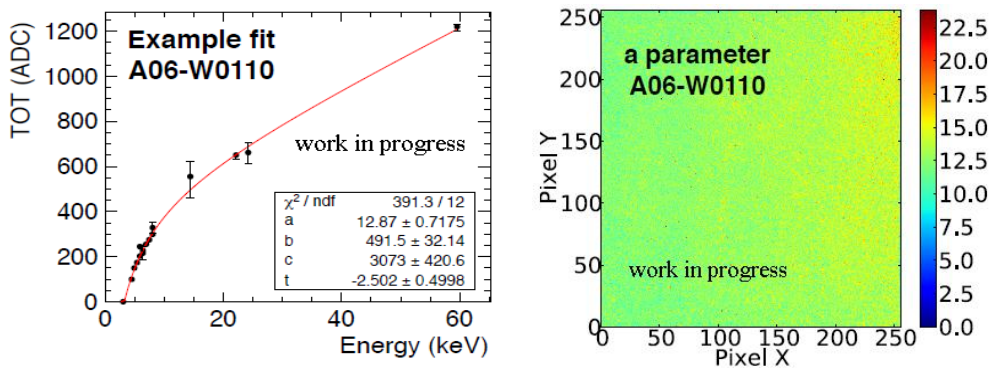

Figure 4: Example of calibration parameters for a Timepix assembly with a $50 \mu \mathrm{m}$ thick sensor. (a): Fit of surrogate response function for one pixel. The surrogate function has four degrees of freedom - $a, b$, $c, t$. We require 5 data points to make the fit. Maps of fitted parameters show assembly uniformity. (b): Value of fit parameter a across the matrix.

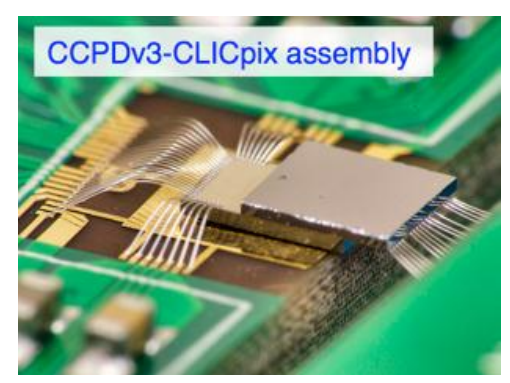

Figure 5: Hybrid glue assembly of a CLICpix prototype chip with a CCPDv3 active sensor on top

\subsection{HV-CMOS + CLICpix assembly}

The CLICpix ASIC has been designed to achieve the requirements of the CLIC vertex detector [6, 7]. This is a fast, low-power chip with a pixel size of $25 \mu \mathrm{m} \times 25 \mu \mathrm{m}$. It is implemented in $65 \mathrm{~nm}$ CMOS technology and provides simultaneous 4-bit time and energy measurements. Power-pulsing and data compression are supported, and it allows for time slicing at the 10 ns level.

This chip was glued to a Capacitive Coupled Pixel Detector (CCPDv3) (see Figure 5). CCPDv3 is an active sensor with two-stage amplifier in each pixel implemented in $180 \mathrm{~nm} \mathrm{HV}$ CMOS process. This allows for the capacitive coupling of the CCPDv3 output pads to the CLICpix input pads through a thin layer of non-conductive glue.

\subsection{HV-CMOS + CLICpix assembly beam tests}

Test-beam experiments with the HV-CMOS + CLICpix assemblies were carried out at the CERN SPS using a beam of positively charged hadrons of $120 \mathrm{GeV}$. The assembly was found to become fully efficient for a threshold of below approximately 1200 electrons. Using the high position resolution for particle tracks, determined by the EUDET telescope, detailed investigations of the pixel response vs. hit position were possible. Details on the test results are given in [8].

\subsection{Power-pulsing, air-flow cooling and mechanical support}

The vertex and tracking detectors at CLIC will be power-pulsed, taking advantage of the bunch train structure of the beams $(50 \mathrm{~Hz}$ operation). In the vertex detector, this allows the dissipated power to remain below $50 \mathrm{~mW} / \mathrm{cm}^{2}$. When the bunch trains collide in the detector, onchip storage capacitors discharge to power the chips. Both analog and digital domains in the chip will be on during collisions. During the $20 \mathrm{~ms}$ gap between bunch trains, the analog electronics will be turned off. Digital electronics will be set to idle, except for readout. Details on the powerpulsing of the CLIC vertex detector can be found in [9].

Despite power-pulsing the vertex detector to minimize the amount of heat dissipated in the inner region, the total heat load which must be removed by the cooling system is around 500 $\mathrm{W}$. It is foreseen to extract this heat using forced air-flow cooling. A spiral endcap geometry on either side of the barrel (see Figure 6) will allow dry air at $0^{\circ} \mathrm{C}$ to be blown through the detector. This has an advantage in terms of material budget, as this cooling system requires no pipes or cooling liquid. Simulations have been performed in order to characterise the air-flow cooling. It was found that a mass flow of $20 \mathrm{~g} / \mathrm{s}$, with an average velocity of $6.3 \mathrm{~m} / \mathrm{s}$ in the barrel, would keep the silicon sensors temperatures below $40^{\circ} \mathrm{C}$. 

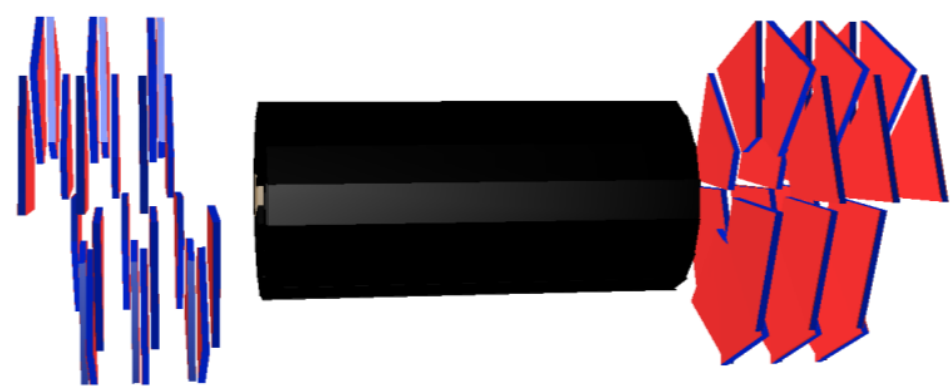

Figure 6: Schematic representation of the vertex detector at CLIC, as presently implemented in the simulations. The central vertex barrel (in black) is $26 \mathrm{~cm}$ long and consists of three double-layers. To allow for efficient air-flow cooling, the end-caps are built as spirals, also forming three double-layers.

The potential danger of air-flow cooling are vibrations induced by the turbulent flow. A dedicated experiment has been performed, with a single vertex layer stave, oriented at three different angles with respect to the flow of air. The resulting vibrations have been measured with laser sensors and the vibration amplitude has been found to be below critical levels (see Figure 7).

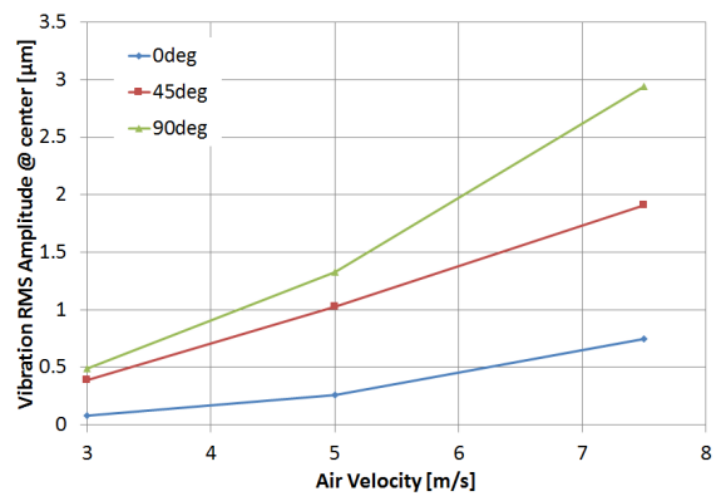

Figure 7: Vibration amplitude in a vertex layer stave as function of air velocity, for different rotation angles.

\section{Tracker simulation studies}

Dense bunches, high energy and small transverse beam size lead to a very high electromagnetic field in the collision region, resulting in beamstrahlung. High occupancies from beaminduced background particles (mostly incoherently produced electron-positron pairs and particles from gamma gamma $\rightarrow$ hadrons interactions) drive small strip sizes for tracking. Full Geant4based detector simulations of the beam-induced background at $3 \mathrm{TeV}$ have been performed. As an example, Figure 8 shows the maximum allowed strip length in the tracker barrel layers, if the maximum allowed occupancy per bunch train is $3 \%$. A strip pitch of $50 \mu \mathrm{m}$, an average cluster size of 2.6 and safety factors of 5 (incoherent pairs) and 2 (gamma gamma $\rightarrow$ hadrons) are assumed. The results of these simulations indicate that the strip lengths in the tracker must be limited to between $2 \mathrm{~mm}$ for the innermost layer to $40 \mathrm{~mm}$ for the outermost one.

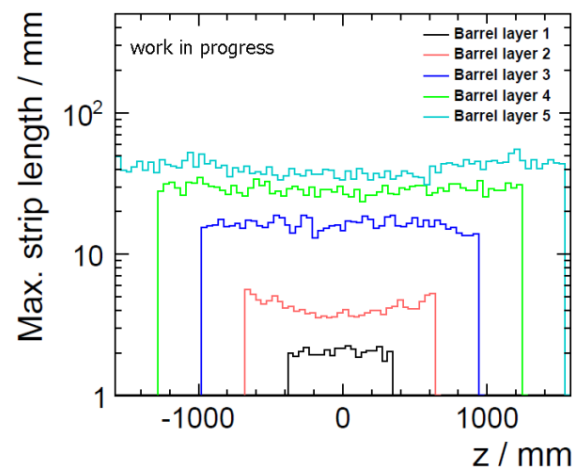

Figure 8: Maximal strip length in the main tracker for a maximum occupancy of $3 \%$ 


\section{Summary}

The CLIC machine environment and the requirements for precision physics measurements place challenging demands on the vertex - and tracking - detector systems. R\&D is being carried out on thin sensors and readout chips, and a comprehensive test beam and lab measurement campaign has demonstrated that thin sensor assemblies with $55 \mu \mathrm{m} \times 55 \mu \mathrm{m}$ pixels can be operated with excellent precision.

The CLICpix ASIC with $25 \mu \mathrm{m} \times 25 \mu \mathrm{m}$ pixels has been designed to provide the necessary features for a readout chip at the CLIC vertex detector, including simultaneous time and energy measurements. It has been tested successfully in AC-coupled assemblies with the CCPDv3 HVCMOS sensor. Powering, cooling and support strategies are being developed, with the objective of minimising the material of the detector.

\section{Acknowledgements}

The author would like to thank: colleagues in the CLICdp collaboration, in particular Sophie Redford and Konrad Elsener for help with preparing these proceeding; Dominik Dannheim, Aharon Levy, Lucie Linssen and Andreas Nurnberg for their valuable comments on the drafts of this paper; Titi Preda for useful discussions. The research leading to these results has received partial funding from the European Commission under the FP7 Research Infrastructures project AIDA, grant agreement no. 262025 .

\section{References}

[1] P. Lebrun et al., The CLIC Programme: Towards a Staged e+e-Linear Collider Exploring the Terascale: CLIC Conceptual Design Report, report CERN 2012-005

[2] D. Henry et al., TSV last for hybrid pixel detectors: Application to particle physics and imaging experiments, Electronic Components and Technology Conference (ECTC), 2013 IEEE 63rd, pp.568, 28-31 May 2013, doi: 10.1109/ECTC.2013.6575630

[3] S. Redford, $R \& D$ for the Vertexing at CLIC, CLICdp-Conf-2014-008

[4] I. Rubinskiy, An EUDET/AIDA Pixel Beam Telescope for Detector Development, Physics Procedia, Volume 37, 2012, Pages 923-931, doi:10.1016/j.phpro.2012.02.434

[5] N. Alipour Tehrani et al., Calibration of ultra-thin hybrid pixel detector assemblies with Timepix readout ASICs, CLICdp-Note-2015-003

[6] S. Redford, CLIC vertex detector R\&D, CLICdp-Conf-2014-003

[7] P. Valerio, R. Ballabriga, M. Campbell, Design of the $65 \mathrm{~nm}$ CLICpix demonstrator chip, LCD-Note-2012-018

[8] N. Alipour Tehrani et al., Capacitively coupled hybrid pixel assemblies for the CLIC vertex detector, CLICdp-Pub-2015-003

[9] G. Blanchot and C. Fuentes, Power pulsing schemes for vertex detectors at CLIC, Journal of Instrumentation, vol. 8, no. 01, p. C01057, 2013. 